\title{
Freeze-out mechanism and phase-space density in ultrarelativistic heavy-ion collisions
}

\author{
Boris Tomášik and Urs Achim Wiedemann \\ CERN, Theory Division, CH-1211 Geneva 23, Switzerland
}

(Received 25 July 2002; published 9 September 2003)

\begin{abstract}
We explore the consequences of a freeze-out criterion for heavy-ion collisions, based on pion escape probabilities from the hot and dense but rapidly expanding collision region. The influence of the expansion and the scattering rate on the escape probability is studied. The temperature dependence of this scattering rate favors a low freeze-out temperature of $\sim 100 \mathrm{MeV}$. In general, our results support freeze-out along finite four-volumes rather than sharp three-dimensional hypersurfaces, with high- $p_{\perp}$ particles decoupling earlier from smaller volumes. We compare our approach to the proposed universal freeze-out criteria using the pion phase-space density and its mean free path.
\end{abstract}

DOI: 10.1103/PhysRevC.68.034905

PACS number(s): 25.75.Dw, 25.75.Ld

\section{INTRODUCTION}

Experiments with nuclear collisions at ultrarelativistic energies are performed with the aim of studying strongly interacting matter under high temperature and density. Such conditions are created very early in the collision. Hadrons at low transverse momentum form the bulk of the particles produced in these collisions. They are produced at the end of the hot and dense partonic phase, but they subsequently scatter in the confined hadronic phase prior to decoupling from the collision system ("freeze-out"). In general, the collective evolution of the hot and dense matter leaves a distinct imprint on the phase-space distribution of the fireball at freezeout. For example, pressure gradients generated in the early stage of the collision and indicative of the equation of state result in collective transverse, radial and elliptic flow which shape important features of hadronic one- and two-particle spectra. However, to disentangle such information from features generated during freeze-out a refined understanding of the decoupling process is needed. This is the condition which has to be satisfied locally for decoupling to take place.

Freeze-out is often modeled as a sudden breakup of the fireball on a fixed three-dimensional hypersurface [1], specified, e.g., by a critical freeze-out temperature or density. This implements in a very simplified way the physical picture that the hadronic scattering rate drops with particle phase-space density and determines freeze-out. However, the difficulties at present in interpreting the HBT radii measured at RHIC [2] motivate to go beyond a sharp three-dimensional implementation of freeze-out. Moreover, the recently observed increase in average pion phase-space density from SPS $(\sqrt{s}$ $=17 \mathrm{~A} \mathrm{GeV})$ to RHIC $(\sqrt{s}=130 \mathrm{~A} \mathrm{GeV})[3,4]$ indicates that - in contrast to an earlier suggestion [5] — additional factors beyond the particle phase-space density must be taken into account. This prompts us in the present work to discuss quantitatively how hadrochemical composition [6,7], local temperature and collective velocity gradients [8], and particle momentum influence the decoupling of particles from the collision region. Since hadrochemistry does change, and freeze-out temperature or velocity gradients may change, in going from SPS to RHIC energies, this study also addresses the possible dependence of hadronic freeze-out on bombarding energy.
Clearly, in the general case, particles decouple from the fireball gradually $[9,10]$, as it is naturally implemented, e.g., in cascade generator simulations. The question arises to what extent this generic behavior can be described by the limiting case of a sharp three-dimensional freeze-out. To discuss this question, we first introduce in Sec. II the so-called escape probability which characterizes the probability of a particle to decouple from the system. In Sec. III, we demonstrate simple examples on how the escape probability is related to the expansion strength and the scattering rate. This illustrates the earlier suggestion [8] that freeze-out occurs when the dilution rate becomes comparable or larger than the scattering rate. Moreover, the scattering rate depends on densities of individual species and the corresponding cross sections $[6,7]$. For pions, e.g., scattering on nucleons is much more important than scattering on other pions due to the larger cross section of the former. In Sec. IV, we discuss the impact of the hadrochemical composition on the scattering rates and freeze-out at SPS and RHIC. This also allows us to address the observed change in the freeze-out phase-space density $[3,4]$. Finally, we turn to the dependence of the scattering rate and the escape probability on temperature. In doing so, we find that $\approx 50 \%$ of the particles are emitted at temperatures below $\sim 100 \mathrm{MeV}$. We discuss this result, together with other findings of our calculations in Sec. V.

\section{THE ESCAPE PROBABILITY}

The following discussion of the freeze-out process $[9,10]$ is based on the particle escape probability $\mathcal{P}(x, p, \tau)$ defined as

$$
f_{\text {esc }}(x, p, \tau)=\mathcal{P}(x, p, \tau) f(x, p, \tau)
$$

Here, $f(x, p, \tau)$ is the distribution function of a given particle species and $f_{\text {esc }}(x, p, \tau)$ denotes the distribution of that fraction of particles which have decoupled from the system prior to time $\tau$ and will not rescatter in the future. For a particle with momentum $p$ at space-time point $(x, \tau)$, the probability to escape the medium without future interaction is $[9,10]$

$$
\mathcal{P}(x, p, \tau)=\exp \left(-\int_{\tau}^{\infty} d \bar{\tau} \mathcal{R}(x+v \bar{\tau}, p)\right) .
$$


Here, the scattering rate $\mathcal{R}(x, p)$ denotes the inverse of the mean time between collisions for a particle at position $x$ with momentum $p$. The opacity integral in Eq. (2) determines the average number of collisions of the particle after time $\tau$. Thus, for a particle which tries to escape the medium at time $\tau$ with velocity $v$, Eq. (2) determines the probability that the particle succeeds in doing so.

The limiting case of a sharp freeze-out along the threedimensional (3D) proper time hypersurface given by $\tau_{\mathrm{fr}}[1]$ can be characterized by an abrupt change of the particle escape probability (2) from 0 to 1 :

$$
\mathcal{P}(x, p, \tau)_{\text {freeze-out }}^{3 \mathrm{D}}=\left\{\begin{array}{lll}
0 & \text { for } & \tau<\tau_{\text {fr }} \\
1 & \text { for } & \tau>\tau_{\text {fr }}
\end{array} .\right.
$$

Freeze-out along more general three-dimensional hypersurfaces [1] can be defined by requiring this kind of threshold behavior of the escape probability along those hypersurfaces. It corresponds to a scattering rate which changes abruptly from a large value to zero along the freeze-out hypersurface.

Hypersurfaces of type (3) are characterized by a criterion which tests only the medium, but does not depend on the particle momentum and particle properties. In the general case of continuous freeze-out, the situation is different since freeze-out does depend on particle momentum and properties. To characterize the four-volume from which particles decouple, one requires [10]

$$
p^{\mu} \partial_{\mu} \mathcal{P}(x, p, \tau)>0 .
$$

This specifies the region in which the amount of escaped particles of momentum $p^{\mu}$ is growing. Note that this general condition also characterizes the freeze-out hypersurface in the case of the sharp freeze-out according to Eq. (3). Condition (4) assumes, however, that there is no additional particle production, i.e., $p^{\mu} \partial_{\mu} f(x, p, \tau)=0$. In the presence of such particle production, one generalizes Eq. (4) to $\partial_{\mu}\left(p^{\mu} \mathcal{P} f\right)$ $>0$.

In general, the evaluation of criterion (4) is complicated. In the Discussion, we shall consider a related, much simpler condition which gives some access to the structure of a fourdimensional freeze-out region on the basis of the escape probability.

\section{THE DEPENDENCE OF PARTICLE FREEZE-OUT ON EXPANSION}

It has been argued a long time ago [8] that freeze-out occurs when the dilution rate becomes larger than the collision rate. Here, we demonstrate how the collision rate and the expansion strength determine the escape probability.

To this end, we consider the simplified case of a particle of vanishing transverse momentum in the center of the fireball. Such a particle does not propagate through layers of different density, but—due to expansion-finds itself in a medium of decreasing density. This simplification allows us to illustrate the effect of dynamical properties of the collision region on freeze-out without being sensitive to further complications of the general case such as finite-size effects which depend on the production point and the velocity of the test particle. We start the discussion of the escape probability (2) by considering a simple power-law ansatz for the scattering rate

$$
\mathcal{R}(p=0, r=0, \tau)=\mathcal{R}(\tau)=\mathcal{R}_{0}\left(\frac{\tau_{0}}{\tau}\right)^{\alpha}, \quad \alpha>1 .
$$

Here, $\mathcal{R}_{0}$ is the scattering rate at the time $\tau_{0}$. Below, we detail the assumptions on which Eq. (5) is based and how the exponent $\alpha$ characterizes the expansion strength of the system. To set the stage, however, we consider first the opacity integral of Eq. (5),

$$
\int_{\tau_{0}}^{\infty} d \tau \mathcal{R}(\tau)=\frac{\mathcal{R}_{0} \tau_{0}}{\alpha-1}
$$

This illustrates the typical interplay of scattering and expansion: a given escape probability can be obtained for different values of the scattering rate, with a higher scattering rate $\mathcal{R}_{0}$ compensated by a stronger expansion (larger $\alpha$ ).

We further illustrate this point with two analytically accessible models for the fireball expansion. Both are based on a factorized ansatz for the scattering rate in terms of the (averaged) cross section $\sigma$ for scattering in the medium, and the average velocity $\bar{v}_{\text {rel }}$ relative to other particles,

$$
\mathcal{R}(\tau)=\sigma \rho(\tau) \bar{v}_{\text {rel }} .
$$

While the time dependence of $\mathcal{R}$ does not factorize in the general case, one may hope to capture the dominant features of a realistic dynamical evolution by retaining the time dependence of density only.

For the first model, we choose a power-law falloff of the density

$$
\rho(\tau)=\rho_{0}\left(\frac{\tau_{0}}{\tau}\right)^{\alpha}
$$

where $\rho_{0}$ is the density at time $\tau_{0}$. From this, expression (5) can be recovered. This time dependence of the density may not be realized during the whole evolution of the fireball; we assume it just in the final stage of the collision. Therefore, time $\tau$ is now defined by the dilution rate $\gamma$,

$$
\gamma \equiv-\frac{1}{\rho} \frac{\partial \rho}{\partial \tau}=\frac{\alpha}{\tau}
$$

Even though we are interested in the situation along the longitudinal symmetry axis of the fireball, time $\tau$ agrees with the "usual" longitudinal proper time $\sqrt{t^{2}-z^{2}}$ only if the density evolves according to Eq. (8) from the very beginning of the collision $(t=0)$. Otherwise it corresponds to a different starting point $t_{0}$ of the time scale ${ }^{1}$

\footnotetext{
${ }^{1}$ Technically, if we were solving for the whole fireball evolution, we would have to match prescription (8) and its first time derivative to the time dependence of the density at earlier times. This is done by tuning $\rho_{0}$ and $\tau_{0}$. Therefore, $\tau_{0}$ has to be a free parameter, and is not necessarily equal to the longitudinal proper time measured from the moment of the first approach of the colliding nuclei.
} 


$$
\tau=\sqrt{\left(t-t_{0}\right)^{2}-z^{2}} .
$$

This is analogous to the Hubble time in cosmology which is defined as the inverse of the expansion velocity gradient. We insert this dynamical information in the usual parametrization of the expansion four-velocity

$$
\begin{aligned}
u^{\mu}= & \left(\cosh \eta \cosh \eta_{t}(\tau, r), \quad \cos \phi \sinh \eta_{t}(\tau, r),\right. \\
& \left.\sin \phi \sinh \eta_{t}(\tau, r), \quad \sinh \eta \cosh \eta_{t}(\tau, r)\right)
\end{aligned}
$$

In accord with relation (10), the space-time rapidity is defined as

$$
\eta=\frac{1}{2} \ln \left[\frac{\left(t-t_{0}\right)+z}{\left(t-t_{0}\right)-z}\right]
$$

and $r, \phi$ are the standard radial coordinates used in the plane transverse to the beam. The transverse rapidity $\eta_{t}$ will be assumed to grow linearly with the radial coordinate. If the particle number is conserved, which is a good assumption at the end of the hadronic phase, the dilution rate is related to the divergence of the velocity field [12]

$$
-\frac{1}{\rho} \frac{\partial \rho}{\partial \tau}=-\frac{1}{\rho} u^{\mu} \partial_{\mu} \rho=\partial_{\mu} u^{\mu}
$$

If we assume that $\eta_{t}(\tau, r) \propto r$, then Eq. (13) links the fourvelocity field (11) with ansatz (8). This specifies the time dependence of the transverse rapidity

$$
\begin{gathered}
\eta_{t}(\tau, r)=\chi \frac{r}{\tau} \\
\chi=\frac{\alpha-1}{2} .
\end{gathered}
$$

In the vicinity of $r=0$, the choice $\chi=1$ leads to a quasiinertial flow which corresponds to an asymptotic solution of the fireball hydrodynamics [13]. The values $\chi<1$ and $\chi>1$ stand for radially decelerating and accelerating flow profiles, respectively. This illustrates the phenomenological consequences of a specific choice of exponent $\alpha$. Relation (15) allows us to rewrite the opacity integral (6)

$$
\int_{\tau_{0}}^{\infty} d \tau \mathcal{R}(\tau)=\frac{\mathcal{R}_{0} \tau_{0}}{\alpha-1}=\frac{\mathcal{R}_{0}}{2}\left(\frac{\chi}{\tau_{0}}\right)^{-1} .
$$

According to Eq. (14), $\chi / \tau_{0}$ is the gradient of transverse rapidity at the time $\tau_{0}$. At $r=0$, it is to a good approximation equal to the transverse velocity gradient. This substantiates the statement made above: a higher scattering rate can be compensated by a stronger transverse flow and still lead to the same escape probability. We note that it is not the average flow velocity, but the local flow gradient which determines the local density decrease and thus enters Eq. (16). In Appendix A we relate this result to the freeze-out criterion, requiring the scattering rate to be smaller than the dilution rate $[8]$.
For a second simple dynamical model of the fireball evolution, we turn to recent hydrodynamic simulations [14]. These indicate that the transverse expansion at the freeze-out stage may be better described by the ansatz

$$
\begin{aligned}
& \eta_{t}(\tau, r)=\xi r, \\
& \xi(\tau)=\mathrm{const},
\end{aligned}
$$

rather than by expressions (8) and (14) used above. In this case, variable $\tau$ is still given by relation (10), but corresponds now to the inverse longitudinal gradient of the expansion velocity:

$$
\tau=\left(\partial_{0} u^{0}+\partial_{3} u^{3}\right)^{-1} .
$$

The ansatz [Eqs. (17) and (18)] implies a different time dependence of the density. From Eq. (13), one finds for the density at the center of the fireball

$$
\rho(\tau)=\rho_{0} \frac{\tau_{0}}{\tau} \exp \left[-2 \xi\left(\tau-\tau_{0}\right)\right]
$$

where $\rho_{0}$ is again the density at time $\tau_{0}$. The corresponding opacity integral reads

$$
\int_{\tau_{0}}^{\infty} d \tau \mathcal{R}(\tau)=\mathcal{R}_{0} \tau_{0} \exp \left(2 \xi \tau_{0}\right) \Gamma\left(0,2 \xi \tau_{0}\right),
$$

where $\Gamma(a, x)$ is the incomplete $\Gamma$ function [15],

$$
\Gamma(a, x)=\int_{x}^{\infty} d t t^{a-1} e^{-t}=\Gamma(a)-\int_{0}^{x} d t t^{a-1} e^{-t} .
$$

Relation (21) is less transparent than Eq. (16), but it shows the same qualitative feature: stronger scattering can be compensated by transverse expansion and can lead to the same escape probability.

\section{THE SCATTERING RATE IN A THERMAL MODEL}

In the preceding section, we studied the effect of expansion on freeze-out. We derived expressions for the opacity integral, which depend on the scattering rate at a fixed time $\tau_{0}$. Here, we calculate this scattering rate and study how it depends on hadrochemistry and temperature. In particular, we determine the scattering rate corresponding to the hadronic final states at SPS and RHIC.

The scattering rate for a test pion of momentum $p$ due to interactions with particles of type $i$ and momentum $k$ can be written as $[16,17]$

$$
\frac{d \mathcal{R}_{i}(x, p)}{d^{3} k}=\rho_{i}(x, k) \sigma_{i}(s) \frac{\sqrt{\left(s-s_{a}\right)\left(s-s_{b}\right)}}{2 \sqrt{m_{\pi}^{2}+p^{2}} \sqrt{m_{i}^{2}+k^{2}}} .
$$

Here, $\sigma_{i}(s)$ denotes the total cross section for collinear collision,

$$
\begin{aligned}
& s_{a}=\left(m_{i}+m_{\pi}\right)^{2}, \\
& s_{b}=\left(m_{i}-m_{\pi}\right)^{2},
\end{aligned}
$$


and the center-of-mass system (c.m.s.) energy $s=s(k, p)$.

We assume that distribution of scattering partners $\rho_{i}(x, k)$ is given by the equilibrium form. In this way, we neglect modification of the distribution function due to decoupling of some particles. Such approximation was argued to cause only a small error in the escape probability in the practically relevant cases [10]. Thus, we write

$$
\rho_{i}(x, k)=\frac{g_{i}}{(2 \pi)^{3}}\left(\exp \left[\left(E_{k}-\mu_{i}\right) / T\right] \pm 1\right)^{-1},
$$

where $g_{i}$ is the degeneracy of the species, $T$ is the temperature, $E_{k}=\sqrt{k^{2}+m_{i}^{2}}$, and the chemical potential $\mu_{i}$ fixes the total density. This is known to provide a good description of the hadronic final state at SPS and RHIC $[18,19]$.

The total scattering rate is obtained by integrating expression (22) over momentum $k$ and summing over all species $i$ :

$$
\mathcal{R}(x, p)=\sum_{i} \int d^{3} k \frac{d \mathcal{R}_{i}(x, p)}{d^{3} k} .
$$

All pion scattering rates are computed in the rest frame of the hadron gas. We include pions, (anti)nucleons, kaons, rhos, and (anti)deltas as scattering partners. The total cross section of pion-baryon scatterings is parametrized as [20]

$$
\begin{aligned}
\sigma(\sqrt{s})= & \sum_{r} \frac{\left\langle j_{i}, m_{i}, j_{\pi}, m_{\pi}|| J_{r}, M_{r}\right\rangle\left(2 S_{r}+1\right)}{\left(2 S_{i}+1\right)\left(2 S_{\pi}+1\right)} \\
& \times \frac{\pi}{p_{\text {c.m.s. }}^{2}} \frac{\Gamma_{r \rightarrow \pi i} \Gamma_{\text {tot }}}{\left(M_{r}-\sqrt{s}\right)^{2}+\Gamma_{\text {tot }}^{2} / 4} .
\end{aligned}
$$

This is the usual Breit-Wigner resonance formula, where $M_{r}$ is the resonance mass and $\Gamma_{\text {tot }}$ and $\Gamma_{r \rightarrow \pi i}$ are the total and the partial width for the given decay channel, respectively. Summation in Eq. (27) runs over all relevant resonance states listed in Ref. [20]. Momentum in the c.m.s. is denoted as $p_{\text {c.m.s. }}$. The prefactor takes care of proper counting of spin and isospin states. The square of the Clebsch-Gordan coefficient $\left\langle j_{i}, m_{i}, j_{\pi}, m_{\pi} \| J_{r}, M_{r}\right\rangle$ assures that the appropriate fraction of the resonance state is picked in the coupling of the isospin states of the two scattering partners. Particle properties are taken from Ref. [21], except for higher excitations with large uncertainties which are taken from Ref. [20]. For scattering on both strange and nonstrange mesons, formula (27) is used again and a 5-mb momentumindependent contribution is added to account for elastic processes. The cross section with baryons is taken to saturate at $30 \mathrm{mb}$ for $\sqrt{s}$ above $3 \mathrm{GeV} / c$. This, however, has a negligible effect on the calculation since high momentum particles are suppressed in the thermal distribution.

To fix the hadrochemical composition of scattering partners at SPS and RHIC, an estimate of the chemical potentials entering Eq. (25) is needed. We take the chemical potential for direct pions from data on pion phase-space densities, and those for all other particle species from ratios of $d N / d y$ at midrapidity. For SPS at $\sqrt{s}=17 \mathrm{~A} \mathrm{GeV}$ and RHIC at $\sqrt{s}$
TABLE I. Chemical potentials in units of MeV used in the calculation for SPS at $\sqrt{s}=17 \mathrm{~A} \mathrm{GeV}$.

\begin{tabular}{cccc}
\hline \hline Temperature & $90(\mathrm{MeV})$ & $100(\mathrm{MeV})$ & $120(\mathrm{MeV})$ \\
\hline$\mu_{p}$ & $477-497$ & $435-455$ & $350-379$ \\
$\mu_{\bar{p}}$ & $301-321$ & $238-259$ & $114-143$ \\
$\mu_{\pi}$ & $50-65$ & $38-53$ & $10-30$ \\
$\mu_{\Delta}$ & $527-562$ & $473-508$ & $360-409$ \\
$\mu_{\bar{\Delta}}$ & $351-386$ & $276-312$ & $124-173$ \\
$\mu_{\rho}$ & $100-130$ & $76-106$ & $20-60$ \\
$\mu_{K}$ & $162-182$ & $132-153$ & $72-102$ \\
$\mu_{\bar{K}}$ & $111-131$ & $76-96$ & $4-33$ \\
\hline \hline
\end{tabular}

$=130 \mathrm{~A} \mathrm{GeV}$, we determine the chemical potentials for three different temperatures: 90, 100, and $120 \mathrm{MeV}$.

In detail, the pion chemical potential at the SPS is obtained by comparing the $m_{\perp}$-dependence of the average phase-space density measured by NA44 [22] and WA98 [23] Collaborations to the result expected from a thermalized boost-invariant source with box transverse density profile and a transverse flow profile $\eta_{t}=\sqrt{2} \eta_{f} r / R_{\text {box }}$ [24]. The transverse momentum spectra relate transverse flow and temperature, such that $\eta_{f} \simeq 0.7$ corresponds to $T$ $=90-100 \mathrm{MeV}$ and $\eta_{f} \simeq 0.55$ for $T=120 \mathrm{MeV}$. From the ratios of $d N / d y$ at midrapidity measured for pions, protons, antiprotons, positive and negative kaons [25,26], particle densities, and corresponding chemical potentials are extracted under the assumption that all particles originate from the same thermal source, in accord with the use of equilibrated distribution in Eq. (22). Resonance decay contribution to pion production is taken into account. The density of neutrons is assumed to be the same as that of protons. Chemical potentials for $\rho$ 's, $\Delta$ 's and $\bar{\Delta}$ 's are deduced by requiring a detailed balance [27]

$$
\mu_{\rho}=2 \mu_{\pi}, \quad \mu_{\Delta}=\mu_{p}+\mu_{\pi}, \quad \mu_{\bar{\Delta}}=\mu_{p}^{-}+\mu_{\pi} .
$$

The chemical potentials extracted in this way are summarized in Table I. The upper and lower values of $\mu$ 's were chosen such that our parameters overpredict or underpredict the data by at least the maximal amount allowed by the quoted error bars. This ensures insensitivity of our conclusions against fine tuning of parameters.

The same procedure is repeated for RHIC at $\sqrt{s}$ $=130 \mathrm{~A} \mathrm{GeV}$. Results are summarized in Table II. The yields at midrapidity for pions, (anti)protons, and kaons were taken from Ref. [28]. Unlike at SPS, the feed-down to (anti)proton yields from weak decays was not corrected for in the used data. We performed a simple correction by assuming that the yields of $\Lambda$ 's and $\Sigma$ 's are given by the same temperature and their chemical potentials are equal to that of the protons. The preliminary data on pion phase-space density presented recently by STAR Collaboration show small statistical error bars only [3], and lie close to the upper bound of the large systematic errors quoted earlier [4]. Our analysis accounts for this not fully clarified experimental situation by associating the central value of the most recent data [3] to the large 
TABLE II. Chemical potentials in units of $\mathrm{MeV}$ used in the calculation for RHIC at $\sqrt{s}=130 \mathrm{~A} \mathrm{GeV}$.

\begin{tabular}{cccc}
\hline \hline Temperature & $90(\mathrm{MeV})$ & $100(\mathrm{MeV})$ & $120(\mathrm{MeV})$ \\
\hline$\mu_{p}$ & $442-489$ & $393-452$ & $291-373$ \\
$\mu_{p}^{-}$ & $407-458$ & $354-418$ & $245-332$ \\
$\mu_{\pi}$ & $78-100$ & $70-97$ & $50-85$ \\
$\mu_{\Delta}$ & $520-589$ & $463-549$ & $341-458$ \\
$\mu_{\bar{\Delta}}$ & $485-558$ & $424-515$ & $295-417$ \\
$\mu_{\rho}$ & $156-200$ & $140-194$ & $100-170$ \\
$\mu_{K}$ & $194-242$ & $170-229$ & $120-202$ \\
$\mu_{\bar{K}}$ & $179-230$ & $153-217$ & $100-187$ \\
\hline \hline
\end{tabular}

(asymmetric) systematic errors of Ref. [4]. To avoid a bias in relating SPS and RHIC energies, we compare the upper bound of the chemical potential for RHIC in Table II (which corresponds to the central value in Ref. [3]) to the upper bound listed in Table I for SPS. We thus underestimate the difference between RHIC and SPS.

Figure 1 shows the corresponding scattering rates for $T$ $=100 \mathrm{MeV}$. The smaller contribution of nucleons at RHIC is largely compensated by antinucleons. Despite the increased pion phase-space density at RHIC, the pionic contribution does not dominate the total scattering rate because of the small pion-pion cross section. Scattering on pions is of comparable importance to scattering on nucleons and antinucleons: both particle species lead to a momentum averaged scattering rate of $\sim 0.2(\mathrm{fm} / \mathrm{c})^{-1}$.

We observe that the increase of pion phase-space density at RHIC when compared to SPS $[3,4]$ has no significant impact on the scattering rate. The earlier suggestion that the pion phase-space density at the freeze-out should be a universal quantity [5] did not account for the contribution of other particle species to the pion scattering rate.

Figure 2 illustrates our results for the various sets of freeze-out temperature and chemical potentials which limit the range of values consistent with data from SPS and RHIC. Although particle densities are approximately the same for all temperatures, the scattering rate increases significantly

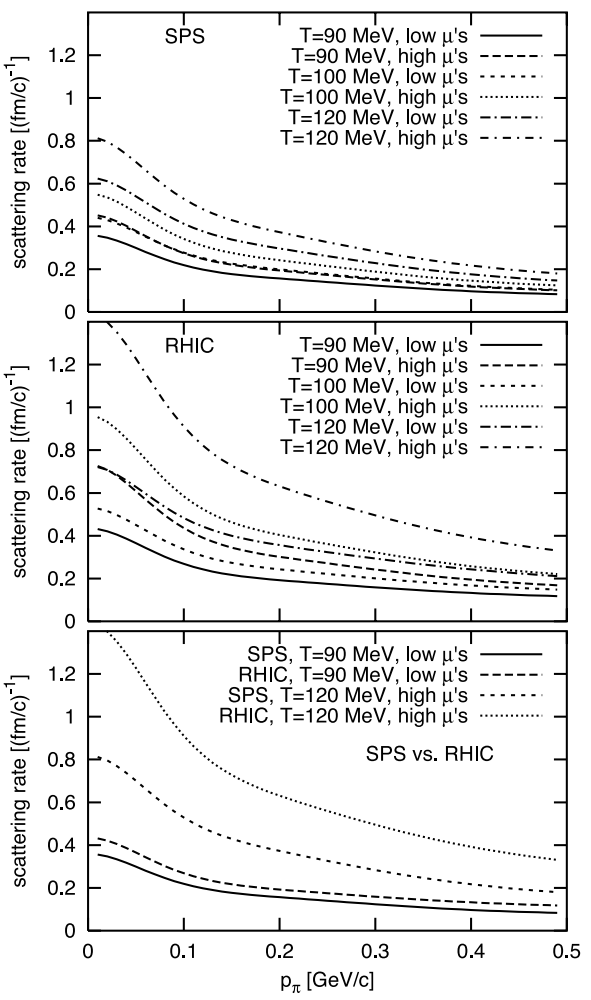

FIG. 2. Pion scattering rate as a function of pion momentum calculated for temperatures 90,100 , and $120 \mathrm{MeV}$ and all corresponding sets of chemical potentials from Tables I (SPS at $\sqrt{s}$ $=17 \mathrm{~A} \mathrm{GeV}$ ) and II (RHIC at $\sqrt{s}=130 \mathrm{~A} \mathrm{GeV})$. The lowest panel summarizes the lowest and the highest curves from both SPS and RHIC.

with temperature. On the other hand, we see a generic decrease of the scattering rate with the pion momentum. This behavior is mainly dictated by phase space available for the collisions, but also depends on the cross section as a function of $s$. We discuss this behavior in more detail in Appendix B.

In the lowest panel of Fig. 2, we compare the extreme results for SPS and RHIC. If the scattering rate in one of the systems was clearly larger than in the other one, this would

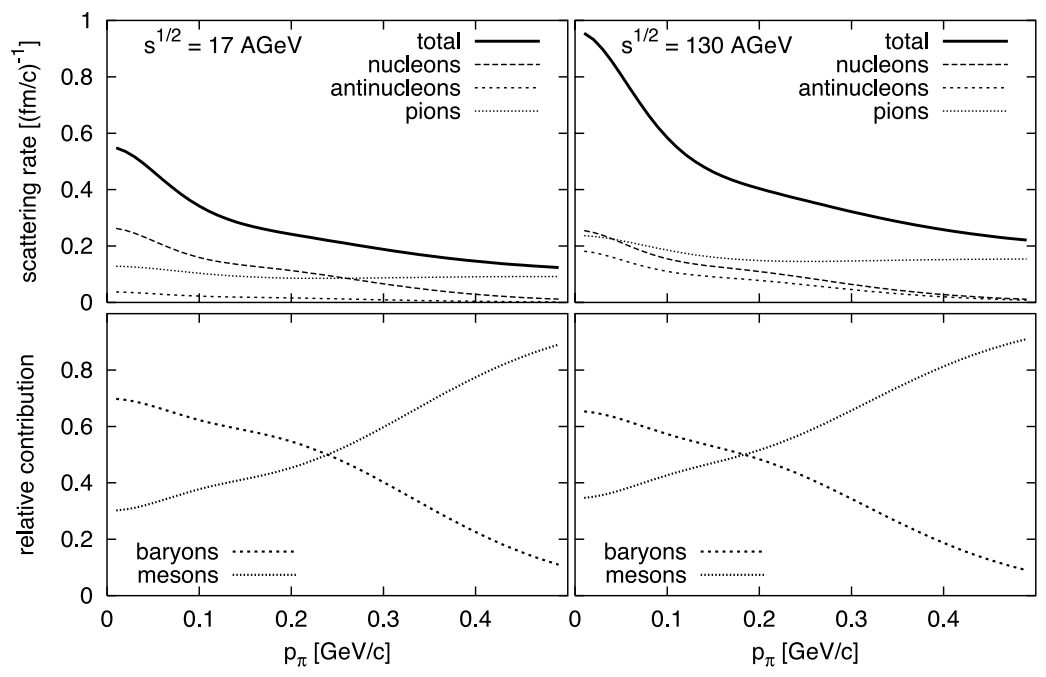

FIG. 1. Pion scattering rate as a function of pion momentum at $T=100 \mathrm{MeV}$ and the highest possible values of chemical potentials for SPS (left column) and RHIC (right column). Contributions to the total scattering rate from scattering on nucleons, antinucleons, and pions are indicated. The lower row shows the baryonic and mesonic relative contributions. 
indicate a stronger transverse expansion in that system, as follows from Sec. III. We see that the present data do not allow to make any conclusion on this subject just by using the scattering rate.

\section{CONCLUSIONS}

In this paper, we have calculated the scattering rates of pions which are characteristic for the late hadronic phase of the collisions at SPS and RHIC. We also illustrated simple examples on how these scattering rates determine the escape probability and how the escape probability is affected by velocity gradients. Moreover, we found that the temperature dependence of the scattering rate allows us to constrain the range of temperatures at which pion emission is significant. To estimate this temperature range, we combined the scattering rates of Sec. IV with the expressions for the opacity integrals (16) and (21). Here, we discuss what is needed to overcome the limitation of our approach and what our results imply for freeze-out in the realistic case.

Going beyond the calculations presented here will require a refined dynamical model which follows the full space-time evolution of the fireball. Most likely, this will involve numerical simulations. In particular, one may hope to extend in this way the expressions for the opacity integral in Sec. III to the case of particles with finite momentum. For a realistic scenario, we envisage the following additional effects:

(i) In a transversely expanding fireball, particles with $p_{\perp}$ $>0$ are produced from parts of the system that comove transversely. Their momentum relative to the medium $p_{\pi}$ is thus smaller than $p_{\perp}$. The corresponding scattering rate $\mathcal{R}\left(p_{\pi}\right)$ is thus higher than the naively expected value at $p_{\perp}$. Hence, this effect lowers the escape probability.

(ii) A particle with finite $p_{\perp}$ can escape the fireball region in a finite time. Thus, the corresponding opacity integral (2) receives a contribution from a finite time only, in contrast to the infinite time which is accumulated by a test particle with $p_{\perp}=0$ in our simplified calculation. This effect leads to a lower value of the opacity integral and enhances the escape probability.

Also, evaluation of the general freeze-out criterion (4) is complicated and requires complete knowledge of the spacetime evolution of the fireball. To gain some understanding of the freeze-out process from the models studied here, however, it is instructive to turn to a simpler condition. Let us define freeze-out for particles at position $x$ with momentum $p$ to occur if the corresponding escape probability $\mathcal{P}(x, p)$ increases above a certain threshold value. Doing this, we actually specify a three-dimensional hypersurface on which a given fraction $f_{\text {esc }} / f=\mathcal{P}$ of all particles is already decoupled. However, in contrast to previous freeze-out criteria [7,5,11], this condition does not only depend on the medium, but also on the particle momentum. Moreover, we can access some properties of the whole four-dimensional freeze-out region by varying the threshold value for $\mathcal{P}$. In this way, the above model calculations give some insight into freeze-out criteria which are more general than the Cooper-Frye type. To put in numbers, a realistic estimate of the transverse flow gradient $\xi=0.08 \mathrm{fm}^{-1}$ and the Bjorken freeze-out time $\tau_{0}=10 \mathrm{fm} / c$ is taken from Ref. [18]. The value $\xi=0.08 \mathrm{fm}^{-1}$ is also in agreement with hydrodynamic simulations [14]. In order to achieve the escape probability of at least $1 / 3$, the opacity integral must be smaller than $-\ln 1 / 3 \approx 1.1$. Then, Eq. (16) leads to a scattering rate of less than $0.18(\mathrm{fm} / c)^{-1}$, while Eq. (21) puts the upper limit for the scattering rate to $0.26(\mathrm{fm} / c)^{-1}$. At a temperature of $120 \mathrm{MeV}$, such a low value of the scattering rate is possibly reached at RHIC for $p_{\pi}>0.4 \mathrm{GeV} / c$ and at SPS at $p_{\pi}>0.3 \mathrm{GeV} / c$, as seen in Fig. 2 . If we increase the required escape probability to $1 / 2$, the scattering rate must be smaller, 0.11 or 0.16 , according to Eqs. (16) and (21), respectively. For particles with $p_{\pi}$ $<0.4 \mathrm{GeV} / c$, the temperature has to drop to $100 \mathrm{MeV}$ if $\mathcal{P}$ should reach $1 / 2$. Particles with $p_{\pi}<0.2 \mathrm{GeV} / c$ must wait even longer. On the other hand, if we put the required value of $\mathcal{P}$ to 0.1 , Eq. (21) leads to an upper bound of $0.54(\mathrm{fm} / c)^{-1}$ for the scattering rate. In the case of $T$ $=120 \mathrm{MeV}$ and high $\mu$ 's at RHIC pions with $p_{\pi}$ $>0.25 \mathrm{GeV} / c$ fulfill this condition; in the less extreme cases, the $10 \%$ escape probability is reached by almost all pions.

This illustrates that particles decouple from the fireball gradually. It indicates that $\approx 10 \%$ of the pions are decoupled at $T=120 \mathrm{MeV}$, but approximately half of them will escape at local temperatures below $100 \mathrm{MeV}$. Thus, a large fraction of the particles decouples at rather low temperatures. We note that a temperature of $120 \mathrm{MeV}$ was assumed as a freeze-out temperature in a recent work [7] where a pion mean free path $\approx 1 \mathrm{fm}$ was suggested as the universal freezeout criterion. This work neglects the temperature dependence of the escape probability and its dependence on the momentum of the particle. Also, it does not consider the possible cancellation between stronger scattering and stronger transverse expansion. In our approach, a thermal distribution of $T=120 \mathrm{MeV}$ at RHIC results in a pion mean free path of only $1.7 \mathrm{fm}$, only slightly larger than that in Ref. [7]. However, only $10 \%$ of all particles are decoupled at this temperature. At lower temperatures, where the fraction of decoupled particles reaches $50 \%$, we find a significantly larger average pion mean free path of at least 3-5 fm.

Let us further comment on the significance of the momentum dependence of the scattering rate. As discussed earlier, at certain $p_{\perp}$ the particle has a momentum with respect to the surrounding medium $p_{\pi}<p_{\perp}$, and thus the $p_{\perp}$-dependence of the scattering rate can be flatter than what is plotted in Fig. 2. We expect, however, that the monotonic increase of the escape probability with $p_{\perp}$ will be robust against refinements of our calculation. This suggests that in contrast to the idealization of a sharp freeze-out along a three-dimensional hypersurface, particle escape is ordered in momentum with high- $p_{\perp}$ particles freezing-out earlier and thus originating from a smaller fireball. This effect could lead to a stronger transverse mass dependence of HBT radii [29] than what is found in current model studies based on a Cooper-Frye freeze-out condition. These latter models (see, e.g., the blastwave model discussed in Ref. [30]) have difficulties in reproducing the observed strong $M_{\perp}$-dependence of HBT radii at RHIC. In what concerns the mechanism proposed here, one 
may envisage compensating effects: for example, if the transverse flow gradient is stronger at later times, then this will reduce the homogeneity regions measured by HBT radii at later times. Such a scenario seems unlikely since it implies acceleration of the collective expansion at a time when particles slowly cease to interact. However, to substantiate this expectation, a model which fully implements the fireball dynamics is needed. In our opinion, this warrants further investigations. In particular, hydrodynamical simulations should be revisited, which are typically based on sharp threedimensional freeze-out and thus miss any contribution from freeze-out along finite four-volumes.

\section{ACKNOWLEDGMENTS}

We thank J. G. Cramer, U. Heinz, J. Pišút, and K. Redlich for fruitful discussions.

\section{APPENDIX A: INTERPLAY OF THE SCATTERING RATE AND THE DILUTION RATE}

Here, we give further details on the relation between the dilution rate and the scattering rate. We start from the opacity integral (6) and we express the exponent $\alpha$ in terms of the dilution rate $\gamma$ via Eq. (9). This leads to

$$
\int_{\tau_{0}}^{\infty} d \tau \mathcal{R}(\tau)=\frac{\mathcal{R}_{0} \tau_{0}}{\alpha-1}=\frac{\mathcal{R}_{0}}{\gamma-\frac{1}{\tau_{0}}} .
$$

This can be directly compared to the statement [8] that freeze-out occurs when the dilution rate is at least as large as the scattering rate. For the scenario of continuous freeze-out considered here, a reasonable escape probability is reached when the opacity integral is of order one or smaller. In the model of Eqs. (5) and (7), this leads to the condition

$$
\gamma-\frac{1}{\tau_{0}} \geq \mathcal{R}_{0},
$$

i.e., there is a factor $-1 / \tau_{0}$ in addition to the standard criterion $\gamma \gtrsim \mathcal{R}_{0}$ [8]. The details of the relation between the scattering rate and the dilution rate at the freeze-out will depend on the particular time evolution of $\mathcal{R}$ and $\rho$, but the feature that larger $\mathcal{R}$ can be compensated by larger $\gamma$ is generic.

\section{APPENDIX B: GENERAL EXPRESSION FOR THE SCATTERING RATE}

In this appendix we derive a general expression for the scattering rate. This allows us to investigate its dependences on temperature and the test particle momentum.

Following Eq. (26) we write the scattering for some particle species of mass $m$. Assuming a Boltzmann distribution of the scattering partners and suppressing the position dependence,

$$
\begin{aligned}
\mathcal{R}(p)= & \int \frac{d^{3} k}{(2 \pi)^{3}} g \exp \left(-\frac{E_{k}-\mu}{T}\right) \\
& \times \sigma(s) \frac{\sqrt{\left(s-s_{a}\right)\left(s-s_{b}\right)}}{2 \sqrt{m_{\pi}^{2}+p^{2}} \sqrt{m^{2}+k^{2}}} .
\end{aligned}
$$

If we change the integration variables to the center-ofmass energy $s$ and the energy of the scattering partner $E_{k}$, and perform the $E_{k}$ integration, this integral leads to

$$
\begin{aligned}
\mathcal{R}(p)= & \frac{g e^{\mu / T}}{8 \pi^{2} p \sqrt{p^{2}+m_{\pi}^{2}}} \\
& \times \int_{\left(m_{\pi}+m\right)^{2}}^{\infty} d s \sigma(s) \sqrt{\left(s-m_{\pi}^{2}-m^{2}\right)^{2}-4 m_{\pi}^{2} m^{2}} \\
& \times \sinh \left(\frac{p}{2 T m_{\pi}^{2}} \sqrt{\left(s-m_{\pi}^{2}-m^{2}\right)^{2}-4 m_{\pi}^{2} m^{2}}\right) \\
& \times \exp \left(-\frac{\sqrt{p^{2}+m_{\pi}^{2}}}{2 T m_{\pi}^{2}}\left(s-m_{\pi}^{2}-m^{2}\right)\right)
\end{aligned}
$$

This scattering rate can be expressed as a convolution of the cross section with the "distribution of two-particle approaches" $\mathcal{D}(p, s)$,

$$
\mathcal{R}(p)=\int_{\left(m_{\pi}+m\right)^{2}}^{\infty} \mathcal{D}(p, s) \sigma(s) d s .
$$

By comparing with Eq. (B3), we obtain

$$
\begin{aligned}
\mathcal{D}(p, s)= & \frac{g e^{\mu / T} \sqrt{\left(s-m_{\pi}^{2}-m^{2}\right)^{2}-4 m_{\pi}^{2} m^{2}}}{8 \pi^{2} p \sqrt{p^{2}+m_{\pi}^{2}}} \\
& \times \sinh \left(\frac{p}{2 T m_{\pi}^{2}} \sqrt{\left(s-m_{\pi}^{2}-m^{2}\right)^{2}-4 m_{\pi}^{2} m^{2}}\right) \\
& \times \exp \left(-\frac{\sqrt{p^{2}+m_{\pi}^{2}}}{2 T m_{\pi}^{2}}\left(s-m_{\pi}^{2}-m^{2}\right)\right) .
\end{aligned}
$$

In the arguments of the exponential functions of Eq. (B8), the momentum of the test particle and the temperature are inversely related. Thus if one ignores the $1 / p \sqrt{p^{2}+m_{\pi}^{2}}$ prefactor, increasing $T$ has the same effect as decreasing $p$. This offers an explanation why $\mathcal{R}$ increases with the raising temperature, but decreases when the momentum becomes larger. There is no collision dynamics going into the calculation of $\mathcal{D}$; it represents merely the phase-space populated with thermally distributed scattering partners. 
However, the temperature and the momentum dependence of the scattering rate is not given solely by the distribution of two-particle approaches, but also reflects details of the convolution of $\mathcal{D}(p, s)$ with the scattering cross section $\sigma(s)$. This can be seen, e.g., by comparing our results with those obtained in Ref. [16]. In that paper, the authors used a simple prescription $\sigma(s) \propto \delta\left(s-M_{r}^{2}\right)$, which leads to a qualitatively different dependence of the scattering rate on the test particle momentum. We verified that this is a consequence of neglecting the width of the resonance. By only assuming the lowest resonance states in a given channel and narrowing the resonance shape, we were able to reproduce the results of Ref. [16] as a limiting case of our calculation.
[1] F. Cooper and G. Frye, Phys. Rev. D 10, 186 (1974).

[2] S. Pratt, Nucl. Phys. A715, 389c (2003).

[3] R.L. Ray, STAR Colaboration, Nucl. Phys. A715, 45c (2003).

[4] J.G. Cramer, lecture delivered at the INT RHIC Winter Workshop 2002 on the "Phase-space density of pions at RHIC," Seattle, WA, USA, 2002 (unpublished); see http:// mocha.phys.washington.edu / int_talk/WorkShops /RHIC02 / People/Cramer_J/

[5] D. Ferenc, U. Heinz, B. Tomášik, U.A. Wiedemann, and J.G. Cramer, Phys. Lett. B 457, 347 (1999).

[6] S. Nagamiya, Phys. Rev. Lett. 49, 1383 (1982).

[7] D. Adamová et al., CERES Collaboration, Phys. Rev. Lett. 90, 022301 (2003).

[8] J. Bondorf, S. Garpman, and J. Zimányi, Nucl. Phys. A296, 320 (1978).

[9] F. Grassi, Y. Hama, and T. Kodama, Z. Phys. C 73, 153 (1996).

[10] Yu.M. Sinyukov, S.V. Akkelin, and Y. Hama, Phys. Rev. Lett. 89, 052301 (2002).

[11] T. Alber et al., NA35 Collaboration, Z. Phys. C 66, 77 (1995).

[12] E. Schnedermann and U.W. Heinz, Phys. Rev. C 47, 1738 (1993).

[13] S.V. Akkelin, T. Csörgő, B. Lukács, Yu.M. Sinyukov, and M. Weiner, Phys. Lett. B 505, 64 (2001).

[14] P.F. Kolb, nucl-th/0304036.

[15] Handbook of Mathematical Functions, edited by M.
Abramowitz and I.A. Stegun (Dover, New York, 1972).

[16] M. Prakash, M. Prakash, R. Venugopalan, and G. Welke, Phys. Rep. 227, 321 (1993).

[17] J. Ftáčnik, P. Lichard, N. Pišútová, and J. Pišút, Z. Phys. C 42, 139 (1989).

[18] B. Tomášik, U.A. Wiedemann, and U.W. Heinz, Heavy Ion Phys. 17, 105 (2003).

[19] T. Csörgö and A. Ster, nucl-th/0207016.

[20] S.A. Bass et al., Prog. Part. Nucl. Phys. 41, 225 (1998).

[21] D.E. Groom et al., Particle Data Group, Eur. Phys. J. C 15, 1 (2000).

[22] M. Murray and B. Holzer, Phys. Rev. C 63, 054901 (2001).

[23] M.M. Aggarwal, WA98 Collaboration, Phys. Rev. C 67, 014906 (2003).

[24] B. Tomášik and U.W. Heinz, Phys. Rev. C 65, 031902 (2002).

[25] J. Bächler et al., NA49 Collaboration, Nucl. Phys. A661, 45 (1999).

[26] S.V. Afanasiev et al., The NA49 Collaboration, Phys. Rev. C 66, 054902 (2002).

[27] H. Bebie, P. Gerber, J.L. Goity, and H. Leutwyler, Nucl. Phys. B378, 95 (1992).

[28] K. Adcox et al., PHENIX Collaboration, Phys. Rev. Lett. 88, 242301 (2002).

[29] F. Grassi, Y. Hama, S.S. Padula, and O.J. Socolowski, Phys. Rev. C 62, 044904 (2000).

[30] B. Tomášik, nucl-th/0304079. 